\title{
Tailoring ORR and HER electrocatalytic performances of gold nanoparticles through metal-ligand interfaces
}

Received 00th January 20xx, Accepted 00th January 20xx DOI: $10.1039 / \times 0 \times x 00000 x$

\author{
David Alba-Molina, ${ }^{a}$ Alain R. Puente Santiago, ${ }^{\text {bc }}$ Juan J. Giner-Casares, ${ }^{* a}$ Enrique Rodríguez-

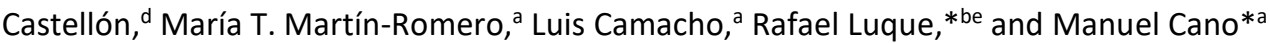

\begin{abstract}
Oxygen reduction (ORR) and hydrogen evolution (HER) reactions are the most important cathodic processes involved on fuel cell and water splitting technologies, respectively. The development of bifunctional electrocatalysts materials plays a key role on the rapid advance of these renewable energy strategies. This work proposes citrate-stabilized gold nanoparticles (AuNPs) as bifunctional electrocatalysts for ORR and HER. The capping ligand shows a great influence on their resulting electrocatalytic performance. A simple ligand exchange method based on concentration gradient has been optimized. The surface structure of the different ligand-stabilized AuNPs was inferred by lead underpotential deposition (Pb-UPD). Static and dynamic electrochemical studies for both ORR and HER have been performed using different ligand-stabilized AuNPs as electrocatalysts, demonstrating that citrate ligand confers the best performance. This work suggests that AuNPs may be suitable as bifunctional electrocatalyst in fuel cells and hydrogen production.
\end{abstract}

\section{Introduction}

Electrocatalysis appears as key process for mass hydrogen production and fuel cell in the essential energy economy for mitigating the climate change and fossil fuels exhaustion. ${ }^{1-4}$ The precise design of bare metallic surfaces in nanostructures is receiving intense attention. ${ }^{5-10}$ Controlling catalytic rates of organic ligand-capped metal nanoparticles through a precise tuning of metal-ligand interfaces is ultimately becoming a fundamental task to design boosted catalytic nanosystems. ${ }^{11-15}$ Traditionally, organic ligands have been closely associated to play an active role over the control of the size and shape of metal nanoparticles as well as their surface properties, such as electron transfer and hot electron cooling processes, ${ }^{16}$ switchable dynamic self-assembly, ${ }^{17}$ metal-support interactions ${ }^{18}$ or colloidal stability. ${ }^{19,20}$ Organic surface ligands have a significant impact on both electronic and steric states of nanostructured systems. The choice of the surface chemistry of nanomaterials modulates their electronic densities and

a. Department of Physical Chemistry and Applied Thermodynamics, University Institute of Nanochemistry (IUNAN), University of Córdoba (UCO), Campus Universitario de Rabanales, Ed. Marie Curie, Córdoba, Spain E-14014. E-mail: jjginer@uco.es; q82calum@uco.es.

b. Department of Organic Chemistry, IUNAN, UCO, Campus Universitario de Rabanales, Edificio Marie Curie, Córdoba, Spain E-14014.E-mail:q62alsor@uco.es. Department of Chemistry, University of Texas at EI Paso, 500 W. University Avenue, El Paso, Texas 79968, United States.

d. Department of Inorganic Chemistry, Crystallography and Mineralogy, Faculty of Sciences University of Málaga, Málaga, Spain.

e.Peoples Friendship University of Russia (RUDN University), 6 Miklukho-Maklaya str., Moscow, Russia.

†Electronic Supplementary Information (ESI) available: FTIR spectra of the pure ligands. Survey and post analysis XPS spectra, DPVs and RDVs curves of the different ligand-stabilized AuNPs. Additional HER analysis by rotating-disc electrode voltammetries measurements and durability tests. See DOI: 10.1039/x0xx00000x reactant accessibilities. ${ }^{21-23}$ Consequently, the rationale design of ligand coating of the electrocatalysts can potentially tune the activity and selectivity of metal nanoparticles in a wide range of catalytic reactions. ${ }^{24-27}$

Organic linkers have recently emerged as promising strategy to increase the catalytic performances of nano-sized structures. Guo et al. stated that the electron-donating properties of phosphine ligands $\left(\mathrm{PPh}_{3}\right)$ magnify the surface electronic density of PdNPs improving the chemoselective catalytic hydrogenation of acetophenone. ${ }^{28}$ In the same direction, Zheng et al. have concluded that interfacial electronic effects provoked by simple organic variations performed on $\mathrm{Pt}$ nanowires can greatly modify their performances towards the catalytic hydrogenation of nitroaromatics. ${ }^{29}$ Additionally, controlling the molecular arrangement of organic modifiers could be changed the strength of the Brønsted acid sites and therefore markedly improve the catalytic efficiency. ${ }^{30}$ In summary, the aforementioned works have opened new horizons to take advantages of the versatility of organic ligands to tune the catalytic function of nanostructured materials in a large variety of reactions.

Despite the tremendous endeavors performed in this emerging field, only a few works have investigated the organic ligands influence applying an electrochemical potential at the metal-ligand interface. Indeed, no reports are available on the effects of organic linkers over the activity of bifunctional nanoparticle electrocatalysts for oxygen reduction (ORR) and hydrogen evolution reactions (HER), which are currently the keystone for the development of renewable-energy technologies. ${ }^{31-33}$ 


\section{Experimental}

\section{Synthesis of AuNPs@Citrate}

$15 \mathrm{~nm}$ citrate-stabilized gold nanoparticles (AuNPs) with a uniform quasi spherical shape and a narrow size distribution were synthesized following a kinetically controlled seeded growth strategy via the reduction of $\mathrm{HAuCl}_{4}$ by sodium citrate. ${ }^{34,35}$ Briefly, $150 \mathrm{~mL}$ of $2.2 \mathrm{mM}$ sodium citrate solution was heated at $\sim 90^{\circ} \mathrm{C}$ in a $250 \mathrm{~mL}$ three-neck round-bottom flask for $15 \mathrm{~min}$ under vigorous stirring. Once the boiling starts, $1 \mathrm{~mL}$ of $25 \mathrm{mM} \mathrm{HAuCl}_{4}$ was injected. After 30 minutes, the reaction was finished. The molar gold concentration of the resulting AuNPs@Citrate was estimated from the absorbance at 400 $\mathrm{nm} \cdot{ }^{36}$

\section{Ligand exchange processes}

Citrate ligand-exchange to CTAB was performed by a simple method based on concentration gradients. For this, $100 \mathrm{mM}$ CTAB solution at $28-30^{\circ} \mathrm{C}$ was added to a previously synthesized AuNPs@Citrate dispersion, with an Au concentration of around $0.7 \mathrm{mM}$, in a ratio of $3: 2 \mathrm{v} / \mathrm{v}$. The resulting mixture was incubated overnight at $28-30{ }^{\circ} \mathrm{C}$ to allow the incorporation of the CTAB ligands on the surface of the AuNPs. Afterwards, the sample was centrifugated at $7500 \mathrm{rpm}$ for 50 minutes. Then, the supernatant was discarded, and the solid was resuspended into a $100 \mathrm{mM}$ CTAB solution at $28-30^{\circ} \mathrm{C}$. After overnight incubation, the sample was centrifugated again at the same conditions. This incubation/centrifugation procedure was performed twice in order to guarantee a complete ligand exchange. Finally, the resulting AuNPs@CTAB were suspended in 10 mM CTAB solution and stored at $28-30{ }^{\circ} \mathrm{C}$ until used.

Citrate ligand-exchange to PSS to obtain AuNPs@PSS was performed using similar protocol described above. The main difference was the used PSS solutions, which had concentrations of $0.4 \mathrm{wt} \%$ and $0.15 \mathrm{wt} \% \mathrm{Na}-\mathrm{PSS}$ ( $\mathrm{Mw}=70 \mathrm{kDa}$ ) for the incubation steps and the storage, respectively.

To perform citrate ligand-exchange to MUA, the most difficult stage was to control the $\mathrm{pH}^{\sim 2}$, both to allow the aqueous solubility of MUA, which requires basic conditions, and to avoid the aggregation of AuNPs. For this, an aqueous solution containing $300 \mathrm{mM}$ MUA and $0.1 \mathrm{M} \mathrm{NaOH}$ was prepared. Once MUA was perfectly dissolved with the help of sonication, the resulting solution was water diluted 1:10, and subsequently added to the previously synthesized AuNPs@Citrate as previously described ligand exchange processes (i.e. in a ratio of $3: 2 \mathrm{v} / \mathrm{v}$ with an $\mathrm{Au}^{\circ}$ concentration of $\sim 0.7 \mathrm{mM}$ ). After overnight incubation at $28-30{ }^{\circ} \mathrm{C}$, the sample was centrifugated at 7500 rpm for $50 \mathrm{~min}$. Then, the supernatant was discarded, and the solid was resuspended into the 1:10 diluted MUA solution (30 $\mathrm{mM}, \mathrm{pH}^{\sim 12}$ ) at $28-30{ }^{\circ} \mathrm{C}$. As described above, the incubation/centrifugation procedure was performed twice in order to guarantee a complete ligand exchange. Finally, the resulting AuNPs@MUA were suspended in 30 mM MUA solution $\left(\mathrm{pH}^{\sim 12}\right)$ and stored at $28-30{ }^{\circ} \mathrm{C}$ until used.

\section{Material characterization}

UV-visible spectra were recorded on a Cary 100 Bio UV-Vis spectrometer in disposable polystyrene cuvettes with $1.0 \mathrm{~cm}$ path length. Samples for transmission electron microscopy
(TEM) were prepared by drying, under ambient conditions, a diluted dispersion of the particles on 200 mesh copper grids coated with Formvar/Carbon film. TEM images were obtained in a JEOL JEM 1400 TEM microscope, operated at an accelerating voltage of $80 \mathrm{kV}$.

XPS studies were performed on a Physical Electronics $\mathrm{PHI}$ 5700 spectrometer (non-monochromatic Mg-Ka radiation, 300 $\mathrm{W}, 15 \mathrm{kV}$ and $1253.6 \mathrm{eV}$ ). Spectra were recorded in the constant pass energy mode at $29.35 \mathrm{eV}$, using a $720 \mu \mathrm{m}$ diameter analysis area. Charge referencing was carried out using the adventitious carbon peak ( $\mathrm{C} 1 \mathrm{~s}$ at $284.8 \mathrm{eV}$ ). The energy scale was calibrated using Cu $2 p 3 / 2, \mathrm{Ag} 3 d 5 / 2$, and $\mathrm{Au} 4 f 7 / 2$ lines at $932.7,368.2$ and $84.0 \mathrm{eV}$, respectively. A PHI ACCESS ESCAV6.0 F and MULTIPAK V9.6 software packages were used for acquisition and data analysis, respectively.

Fourier-transform infrared (FTIR) spectroscopy was performed in an ALPHA-T Bruker spectrometer. Spectra were recorded from dried-samples diluted in $\mathrm{KBr}$, at room temperature in a $4000-600 \mathrm{~cm}^{-1}$ wavenumber range, using the OPUS software.

\section{Electrochemical experiments}

Cyclic (CVs) and differential pulse (DPVs) voltammetric measurements were recorded on an AUTOLAB PGSTAT30 electrochemical analyzer using a three-electrode system. A glassy carbon (GC) disc (5 $\mathrm{mm}$ in diameter; Pine Instruments Company) were used as working electrode. A drop of $25 \mu \mathrm{L}$ of AuNPs sample $\left(\mathrm{Au}^{\circ}\right.$-concentration $\sim 0.7 \mathrm{mM}$ ) was loaded onto the clean surface of GC electrode and then dried overnight at 4 ㅇ. . A platinum sheet and an $\mathrm{Ag} / \mathrm{AgCl}$ electrode were used as counter and reference electrodes, respectively. $0.5 \mathrm{M} \mathrm{KOH} \mathrm{O}_{2}-$ saturated and $0.5 \mathrm{M} \mathrm{H}_{2} \mathrm{SO}_{4} \mathrm{~N}_{2}$-saturated solutions were used as supporting electrolytes for ORR and HER, respectively.

For the HER measurements, all the potentials versus the $\mathrm{Ag} / \mathrm{AgCl}(3 \mathrm{M} \mathrm{KCl})$ reference electrode were converted to the reversible hydrogen electrode (RHE) scale using the Nernst equation: ${ }^{37}$

$$
E_{R H E}=E_{A g / A g C l}+0.059 p H+E_{A g / A g C l}^{\theta}
$$

Where $E_{R H E}$ is the converted potential versus $\mathrm{RHE}, E_{A g / A g C l}$ is the experimental potential measured against the $\mathrm{Ag} / \mathrm{AgCl}$ reference electrode, and $\mathrm{E}_{\mathrm{Ag} / \mathrm{AgCl}}$ is the standard potential of $\mathrm{Ag} / \mathrm{AgCl}(3 \mathrm{M} \mathrm{KCl})$ at $25^{\circ} \mathrm{C}(0.205 \mathrm{~V})$. The HER electrochemical measurements were carried out in $0.5 \mathrm{M} \mathrm{H}_{2} \mathrm{SO}_{4}(\mathrm{pH}=0.29)$ at room temperature: therefore, $E_{R H E}=E_{A g / A g C l}+0.222 \mathrm{~V}$.

\section{Results and discussion,}

\section{Ligand exchange characterization}

Stable aqueous dispersions of AuNPs@CTAB, AuNPs@PSS and AuNPs@MUA were successfully prepared from AuNPs@Citrate through simple ligand-exchange processes based on concentration gradient. To demonstrate the efficient functionalization of AuNPs with different capping ligands, UVvisible, Fourier-transmission infrared (FTIR), transmission electron microscopy (TEM) and X-ray photoelectron spectroscopy (XPS) analysis were performed. 

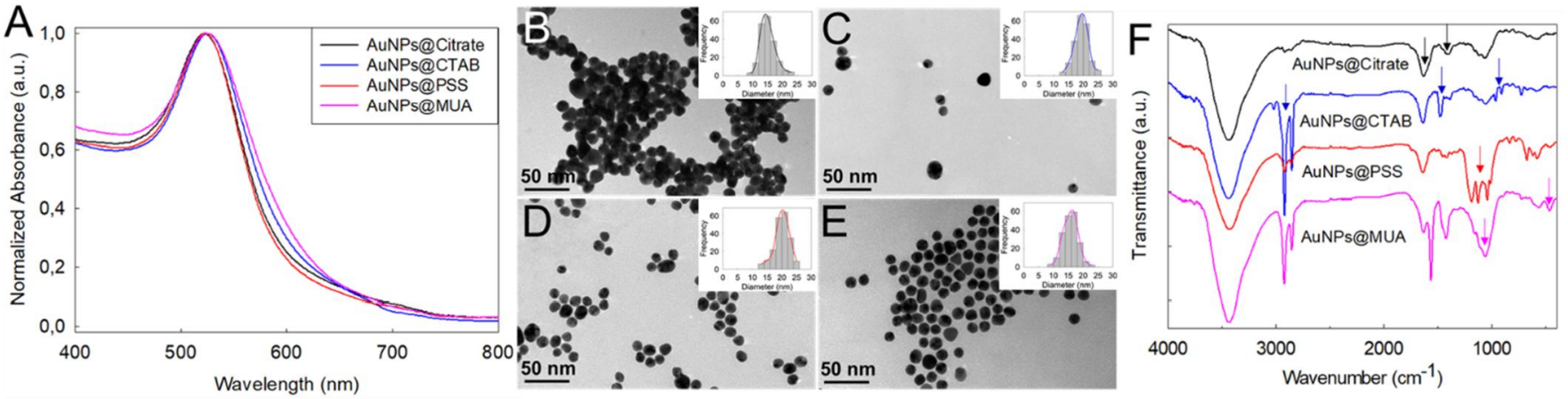

Fig. 1 (A) UV-visible spectra of the different ligand-stabilized AuNPs. (B-E) TEM images of AuNPs@Citrate, AuNPs@CTAB, AuNPs@PSS and AuNPs@MUA, respectively. Inset their size distribution histograms. (F) FTIR spectra of all these AuNPs.

Fig. 1A shows the normalized UV-vis spectra for the ligandstabilized AuNPs. Slight variations on the broadening of the surface plasmon resonance (SPR) band related to the different electronic interactions between Au-core and the surface capping ligands were observed. The SPR band maxima $\left(\lambda_{\max }=\right.$ $521 \mathrm{~nm}$ ) did not show a significant change after the ligandexchange processes, suggesting good preservation of the Au core. TEM images of the different ligand-stabilized AuNPs further confirmed the absence of significative changes both on size distribution and morphological parameters, see Fig. 1B-E.

The molecular species associated with the obtained AuNPs from the reported ligand-exchange processes could be identified by FTIR analysis (Fig. 1F). AuNPs@Citrate showed the expected carboxylate bands of the citrate coating, such as the asymmetric $\mathrm{C}=\mathrm{O}\left(1637 \mathrm{~cm}^{-1}\right)$ and symmetric C-O $\left(1408 \mathrm{~cm}^{-1}\right)$ stretching bands. ${ }^{35,38}$ The presence of CTAB on the AuNPs@CTAB was verified by strong bands associated with $\mathrm{sp}^{3}$ $\mathrm{C}-\mathrm{H}$ stretching $\left(2800-2900 \mathrm{~cm}^{-1}\right),-\mathrm{CH}_{3}$ deformation $\left(1477 \mathrm{~cm}^{-1}\right)$, and $\mathrm{C}-\mathrm{N}$ stretching $\left(920\right.$ and $\left.966 \mathrm{~cm}^{-1}\right) .39$ While citrate ligandexchange to PSS, AuNPs@PSS showed additional peaks associated with the $-\mathrm{SO}_{3}{ }^{-}$groups $\left(1010-1220 \mathrm{~cm}^{-1}\right) \cdot{ }^{39}$ Finally, after exchange with MUA, the FTIR spectrum of AuNPs@MUA exhibited a typical COO stretch at $1637 \mathrm{~cm}^{-1}$, which red-shifted from the $\mathrm{COOH}$ stretch in MUA $\left(1690 \mathrm{~cm}^{-1}\right.$ in Fig. S1) due to the formation of $\mathrm{H}$-bonds between neighboring carboxylic acids. In addition, the lack of S-H stretching band $\left(2520-2700 \mathrm{~cm}^{-1}\right.$ in pure MUA, Fig. S1) and the appearance of a C-S stretch at $\approx 580$ $\mathrm{cm}^{-1}$ confirmed both the presence of MUA and the formation of Au-S bonds. ${ }^{40,41}$

XPS measurements not only assisted in further confirming the ligand-exchange procedure, but also offered insights on the oxidation state of Au-surface. Fig. 2 plots the survey spectra of the different ligand-stabilized AuNPs on ITO supports, showing the presence of $\mathrm{Au} 4 f, \mathrm{C} 1 s$ and $\mathrm{Au} 4 d$ in all samples. As expected, $\mathrm{Na} 1 \mathrm{~s}$ and $\mathrm{O} 1 \mathrm{~s}$ were detected in all AuNPs, except in the case of AuNPs@CTAB. On the other hand, S $2 p$ and S $2 s$ were only detected in AuNPs@PSS and AuNPs@MUA samples displaying significant differences between both signals, while $\mathrm{N}$ $1 s, \mathrm{Br} 3 p(180 \mathrm{eV})$ and $\mathrm{Br} 3 d(68 \mathrm{eV})$ were only detected in AuNPs@CTAB. ${ }^{41}$

Fig. 2A compares in greater detail the high-resolution $\mathrm{N} 1 \mathrm{~s}$ core level spectrum of AuNPs@Citrate and AuNPs@CTAB, showing a single contribution at $402 \mathrm{eV}$ typical of an alkylammonium cation. ${ }^{39}$ The deconvoluted $S 2 p$ core level spectra of S-containing AuNPs@PSS and AuNPs@MUA show the typical doublet $S 2 p_{3 / 2}$ and $S 2 p_{1 / 2}$. The peaks at 168.4 and $169.6 \mathrm{eV}$ were assigned to the sulfonic group on the Au surface of AuNPs@PSS (Fig. 2C), whereas the main doublet at 163.2 and 164.4 eV was assigned to thiolated AuNPs in AuNPs@MUA (Fig. 2D). ${ }^{41}$ Additional contributions with comparatively much lower relative intensity were assigned to sulfide groups at low binding.

To gain further insights in Au binding energies associated with distinct capping ligands, high-resolution XPS spectra of $\mathrm{Au}$ $4 f$ were compared in Fig. 3A. Significant shifts on the Au $4 f$ states of the different decorated AuNPs can be observed, which can be attributes to changes in their interfacial composition due to the influence of the surface ligands. Fig. 3B-D show the deconvoluted high resolution $\mathrm{Au} 4 f$ core level spectra for AuNPs@CTAB, AuNPs@PSS and AuNPs@MUA, respectively. The $A u$ if signal for AuNPs@Citrate did not require deconvolution, where the peaks at $83.1 \mathrm{eV}$ and $86.7 \mathrm{eV}$ were assigned to $A u 4 f_{7 / 2}$ and $A u 4 f_{5 / 2}$, respectively, belonging both photoemissions to $\mathrm{Au}(0) .^{41,42}$ Intriguingly, the $A u 4 f_{7 / 2}$ binding energy $(83.1 \mathrm{eV})$ was clearly shifted to lower value energy compared with the same signature reported for a pure metallic $\mathrm{Au}$, which was attributed to the negatively surface charged AuNPs@Citrate. ${ }^{43}$ The Au-CTAB interaction also provided the appearance of two doublets, where two contributions of $\mathrm{Au}$ $4 f_{7 / 2}$ appear at $82.4 \mathrm{eV}(17 \%)$ and $84.1 \mathrm{eV}(83 \%)$, respectively (Fig. 2B). The low binding energy signal was assigned to the positively charged AuNPs@CTAB whilst the second one, much more intense, to $A u$ interacting with bromide ion. For AuNPs@PSS (Fig. 3C), the Au $4 f$ spectrum also showed two doublets, but two contributions of Au $4 f_{7 / 2}$ located at $83.7 \mathrm{eV}$ (92\%) and $85.6 \mathrm{eV}(8 \%)$, respectively. The main signal was assigned to $\mathrm{Au}$ interacting with sulfonic group and the weaker one to metallic Au. Fig. 3D shows the deconvoluted spectrum for AuNPs@MUA with two doublets, with the Au 4f $f_{7 / 2}$ signals at $82.4 \mathrm{eV}(39 \%)$ and $84.3 \mathrm{eV}(61 \%)$, respectively. Although the resulting binding energy values of the $A u 4 f$ core level were quite similar to the one obtained for AuNPs@CTAB, the relative intensities for the latter presented more intense signal probably due to the high binding energy of the Au-S interaction.

The variations in Au binding energies of the different ligandstabilized AuNPs and the influence on their surface structure and the ratio of the existing facets were further investigated by lead underpotential deposition (Pb-UPD). Typically, during the positive potential sweep, three stripping peaks corresponding 
to the lead layer desorption on the Au (111), (100) and (110) respectively.44,45 Fig. 4A shows that AuNPs@Citrate mostly facets could be observed at around $-0.7,-0.57$, and $-0.47 \mathrm{~V}$, presents (100) symmetry whilst the surface sites of
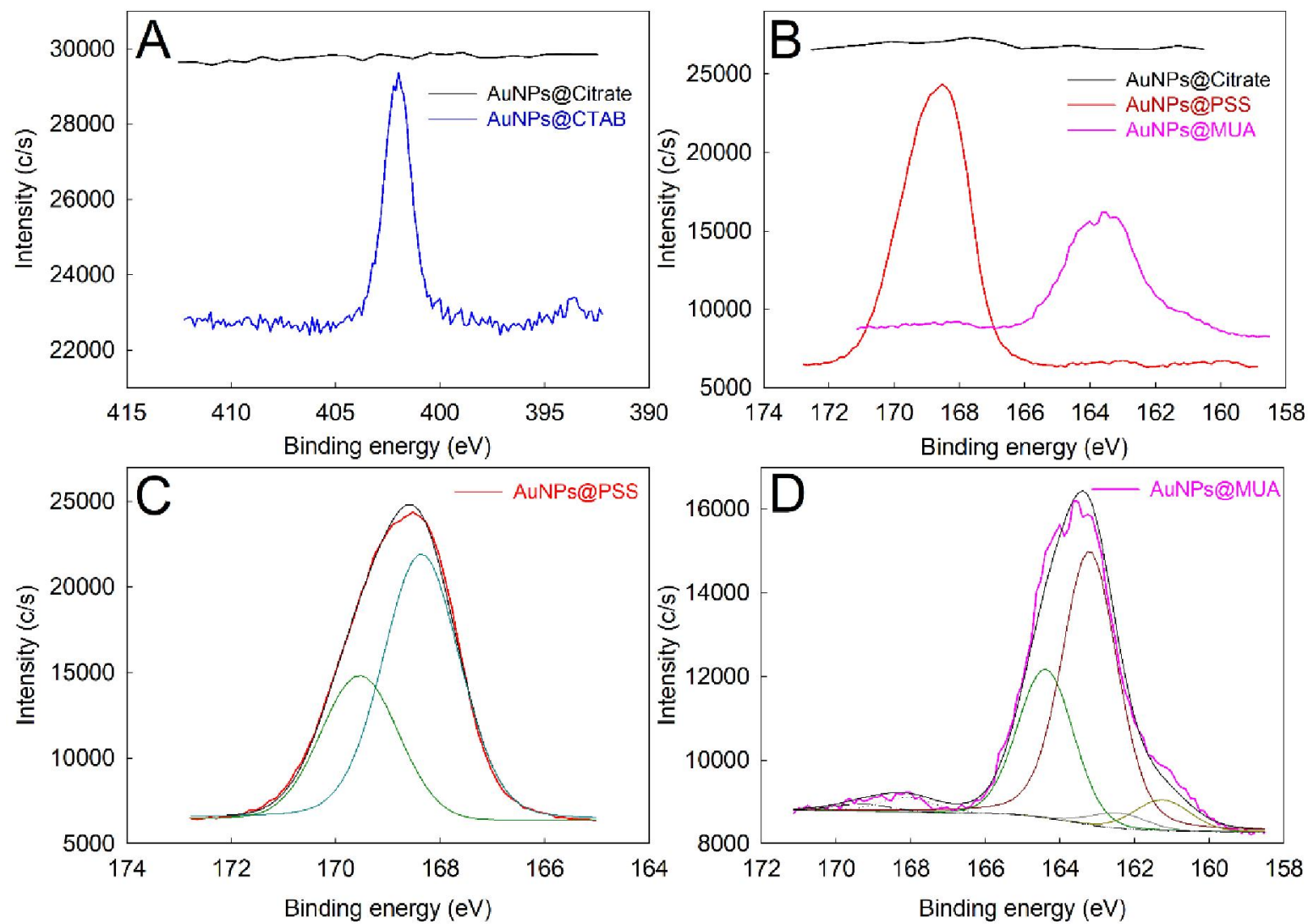

Fig. 2 High-resolution XPS spectra of N 1s for AuNPs@Citrate and AuNPs@CTAB (A), and of S 2p for AuNPs@Citrate, AuNPs@PSS and AuNPs@MUA (B). The deconvoluted high-resolution S $2 p$ core level spectra for AuNPs@PSS (C) and AuNPs@MUA (D).
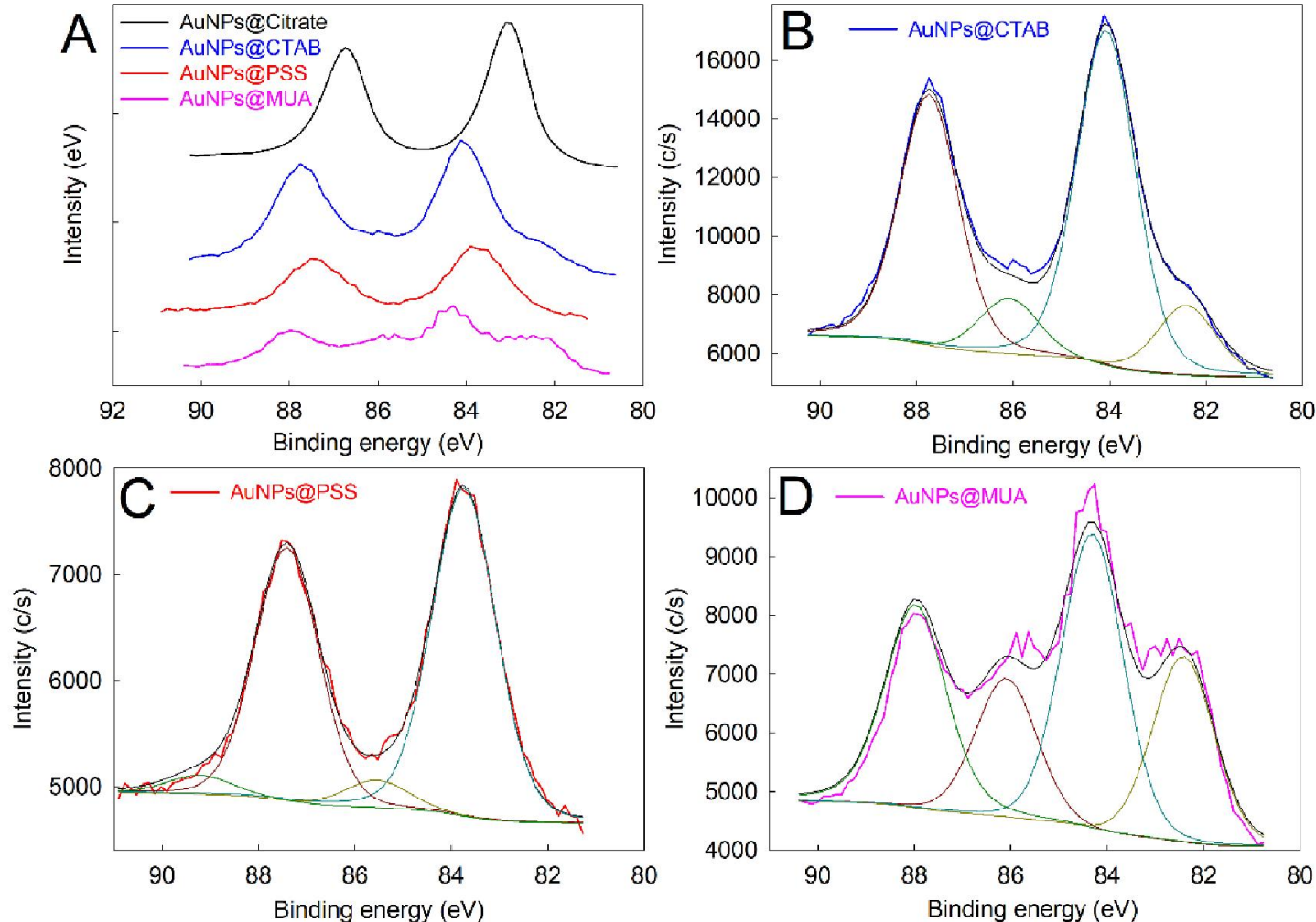

Fig. 3 High-resolution XPS spectra of Au $4 f$ for the different ligand-stabilized AuNPs (A), and the deconvoluted high-resolution Au $4 f$ core level spectra for AuNPs@CTAB (B), AuNPs@PSS (C) and AuNPs@MUA (D). 
AuNPs@CTAB, AuNPs@PSS and AuNPs@MUA display (111) domains but with two peaks of different relative intensities and widths. The latter electrochemical analysis supports our hypothesis that binding energy shifts were associated with variations on the surface structure of the AuNPs due to the influence of the capping ligands. ${ }^{46}$ Although the $\mathrm{Au}(111)$ surface is the most populated facet due to having the lowest surface energy, our results for AuNPs@Citrate agreed with Park et al., demonstrating that citrate chains also fit to the surfaces of $\mathrm{Au}(100)$ and $\mathrm{Au}(110)$ with the same configuration of van der Waals and hydrogen bond interactions that $\mathrm{Au}(111)$ facets. ${ }^{38}$ The obtained surface structure for AuNPs@CTAB was surprising, given that CTAB shows preferential adsorption on gold facets as: $(100) \approx(110)>(111) .{ }^{47}$ Concerning AuNPs@MUA, Kumar et al. proposed that thiol derivatives preferentially bind onto the (111) planes because they provide the lowest surface energy among other facets, ${ }^{48}$ whereas Chen et al. concluded the following sequence for thiol adsorption at different sites: corner $>$ edge $>(100) \sim(111)$ facets. ${ }^{49}$ No reports are available for AuNPs@PSS to the best of our knowledge. According to our results PSS seemed to present preferential adsorption by facets (111).

\section{ORR analysis}

Static and dynamic electrochemical studies were performed using the ORR as model to investigate the influence of the Au-
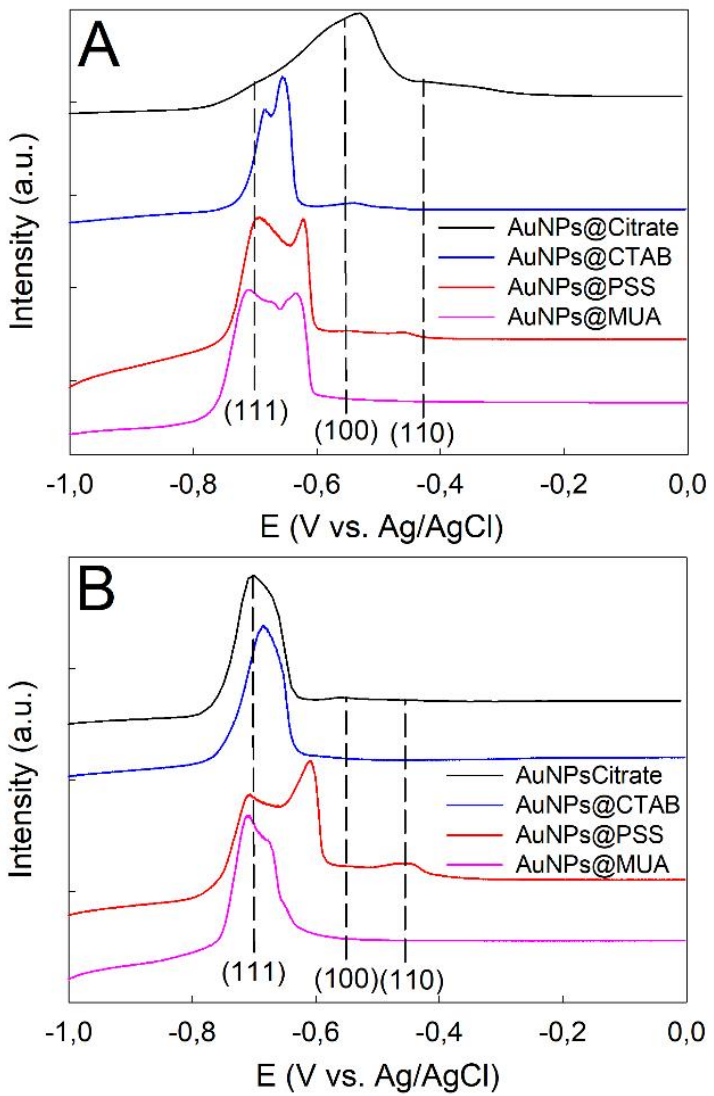

Fig. 4 Desorption voltammetric profiles of the different ligand-stabilized AuNPs, before (A) and after (B) ORR measurements, in $0.1 \mathrm{M} \mathrm{NaOH}$ and 1 $\mathrm{mM} \mathrm{Pb}\left(\mathrm{NO}_{3}\right)_{2}$ recorded under $\mathrm{N}_{2}$-saturated recorded at $20 \mathrm{mV} \cdot \mathrm{s}^{-1}$. ligand interface on their electrocatalytic properties. Fig. 5A shows the electrochemical response of the Au-core under $\mathrm{N}_{2-}$ saturared conditions. AuNPs@MUA and AuNPs@PSS do not show the typical Au/AuOx redox process associated with AuNPs due to both steric and electronic effects of these capping ligands, which passivate the Au-surface. While the cathodic peak maximum of AuNPs@CTAB was located at $-0.05 \mathrm{~V}$ that is approximately $0.1 \mathrm{~V}$ negatively shifted regarding AuNPs@Citrate. Fig.5B includes the electrocatalytic effect of AuNPs in oxygen reduction processes. It should be noted that the intensities of the cathodic peaks as well as the onset potential of the $\mathrm{O}_{2}$ reduction varied significantly with the capping ligands, demonstrating a clear dependence of the
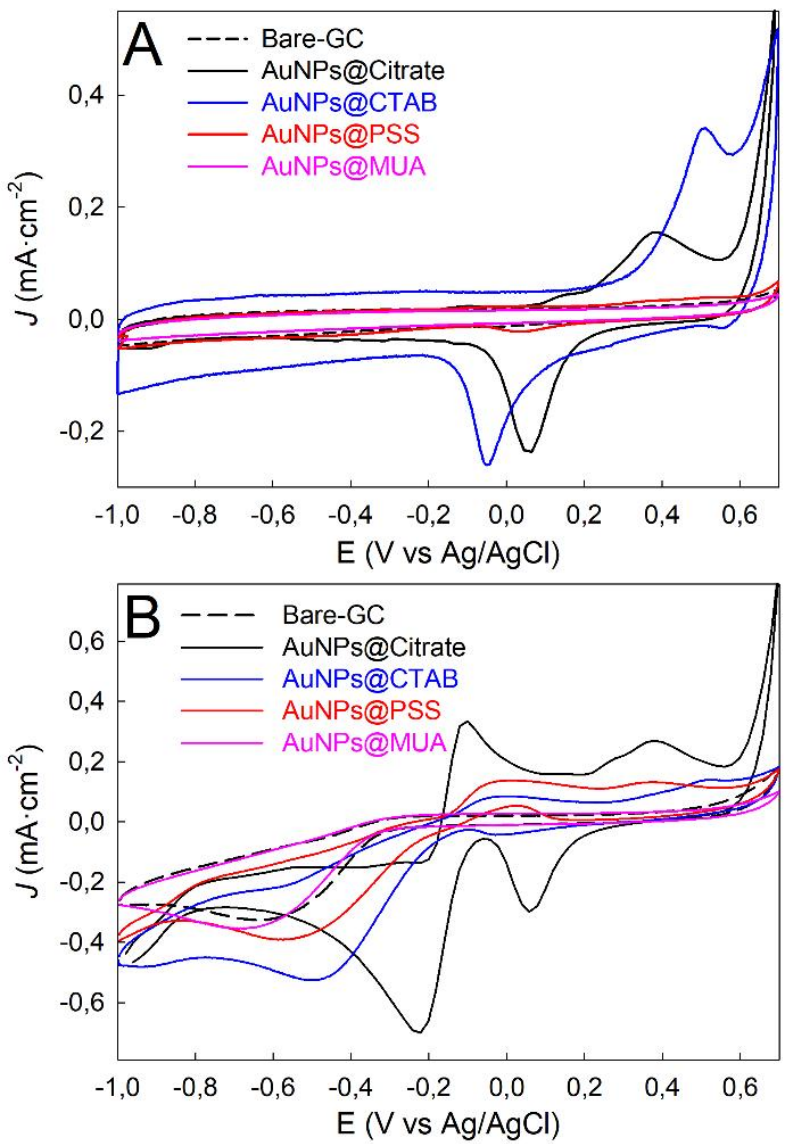

Fig. $5 \mathrm{CV}$ s curves obtained for GC electrodes modified with different ligand-stabilized AuNPs in $\mathrm{N}_{2}$-saturated (A) and $\mathrm{O}_{2}$-saturated (B) $0.5 \mathrm{M}$ $\mathrm{KOH}$ at $0.1 \mathrm{~V} \cdot \mathrm{s}^{-1}$.

metal-ligand interface. This observation is consistent with the DPVs measurements (Fig. S3).

Further insight of the ligand-capping effect on the electrocatalytic activity of AuNPs towards ORR were provided by analyzing the reaction kinetics properties using rotating-disk electrode voltammetry (RDV). A significant hysteresis between the initial forward- and the return-scan attributed to the different interaction between $\mathrm{Au}$ surfaces and dioxygen molecules was found, see Fig. $\$ 4 .{ }^{45}$ Hysteresis has been reported to depend on several factors, such as: particle size, amount of loaded sample and applied potential range. We provided herein experimental evidence on the dependence of 
hysteresis with the Au-ligand interface. The physicochemical process leading to the observed hysteresis is the displacement of the capping ligand required for the dioxygen adsorption during the initial forward scan, which is highly dependent on the ligand type and bonding interaction with gold surface. Moreover, secondly, the limited current density at $-1.0 \mathrm{~V}$ using the return scan for the kinetic analysis varies significatively according to the following order: AuNPs@Citrate > AuNPs@CTAB > AuNPs@PSS > AuNPs@MUA, obtaining values of $-5.58,-3.64,-3.15$, and $-2.67 \mathrm{~mA} \cdot \mathrm{cm}^{-2}$, respectively (Fig. $6 \mathrm{~A}$ ). The onset potential values were quite similar for the different ligand-stabilized AuNPs (Table 1). The passivation both the AuNPs and GC surfaces was confirmed by the lower resulting maximum current density at -1.00 V for AuNPs@MUA than for bare-GC. The striking influence of the ligand coating on the ORR electrocatalytic performance was revealed by the comparatively higher differences in the obtained intensity values for AuNPs with different capping ligands than for varying size of the AuNPs, e. g., from 15 to $95 \mathrm{~nm} .{ }^{45}$

Table 1 summaries the kinetic parameters at $-1.00 \mathrm{~V}$ for the different ligand-stabilized AuNPs obtained from Plots in Fig. S5 by applying the Koutecky-Levich (K-L) equations:

$$
\frac{1}{J}=\frac{1}{J_{L}}+\frac{1}{J_{K}}=\frac{1}{B \omega^{1 / 2}}+\frac{1}{J_{K}}
$$
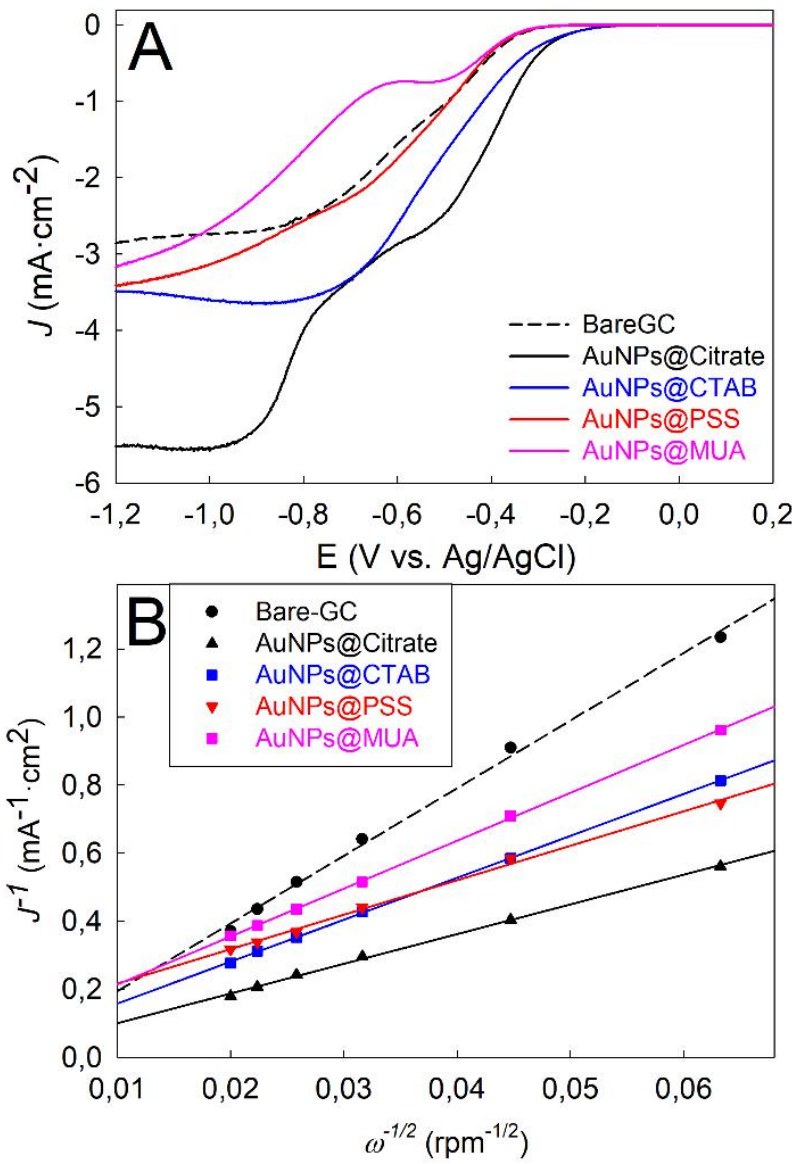

Fig. 6 (A) RDVs curves of GC electrodes modified with different ligandstabilized AuNPs at same rotating rate of $2500 \mathrm{rpm}$. Scan rate: $10 \mathrm{mV} \cdot \mathrm{s}^{-1}$ (B) Koutecky-Levich plots obtained from the RDVs in Fig. S4 at $-1.00 \mathrm{~V}$.

$$
J_{K}=n F k C_{0}
$$

where $J$ is the measured current density, $J_{K}$ and $J_{L}$ are the kinetic and diffusion limiting current densities, respectively, $\omega$ is the electrode rotation rate, $n$ is the overall number of electrons transferred in oxygen reduction, $F$ is the Faraday constant, $C_{0}$ is the bulk concentration of $\mathrm{O}_{2}$ dissolved in the electrolyte $\left(1.03 \cdot 10^{-3} \mathrm{~mol} \cdot \mathrm{L}^{-1}\right.$ for $\left.0.5 \mathrm{M} \mathrm{KOH}\right), D_{0}$ is the diffusion coefficient of $\mathrm{O}_{2}\left(1.63 \cdot 10^{-5} \mathrm{~cm}^{2} \cdot \mathrm{s}^{-1}\right.$ for $\left.0.5 \mathrm{M} \mathrm{KOH}\right), v$ is the kinematic viscosity of the electrolyte $\left(0.01 \mathrm{~cm}^{2} \cdot \mathrm{s}^{-1}\right.$ for $\left.0.5 \mathrm{M} \mathrm{KOH}\right)$, and $k$ is the electron transfer rate constant during ORR. ${ }^{45} B^{*}$ is a constant $\left(2.76 \cdot 10^{-2} \mathrm{~A} \cdot \mathrm{cm}^{-2} \cdot \mathrm{rpm}^{-1 / 2}\right)$ that is the same for all the performed experiments. Fig. $6 \mathrm{~B}$ shows the resulting $\mathrm{K}-\mathrm{L}$ plots for all AuNPs samples, demonstrating first-order reaction kinetics toward dissolved oxygen due to the excellent linearity of the experimental points. Overall, the obtained values for $n, J_{k}$ and $k$ during ORR decrease as a function of the capping ligand in the following order: AuNPs@Citrate > AuNPs@PSS AuNPs@CTAB $>$ AuNPs@MUA. Therefore, AuNPs coated with citrate ligand revealed as highly efficient electrocatalysts, whereas coating of the AuNPs with MUA ligands significantly decreased the electrocatalytic performance.

$$
B=0.62 n F C_{0} D_{0}^{2 / 3} v^{1 / 6}=B^{*} n
$$

Table 1. Kinetic parameters for ORR of the GC electrodes modified with

\begin{tabular}{|c|c|c|c|c|c|}
\hline $\begin{array}{l}\text { ORR Kinetic } \\
\text { parameters } \\
\text { at }-1.00 \mathrm{~V}\end{array}$ & AuNPs@Citrate & AuNPs@CTAB & AuNPs@PSS & $\begin{array}{c}\text { AuNPs@ } \\
\text { MUA }\end{array}$ & $\begin{array}{c}\text { Bare- } \\
\text { GC }\end{array}$ \\
\hline $\begin{array}{c}\text { Onset } \\
\text { potential (V) }\end{array}$ & -0.20 & -0.20 & -0.24 & -0.24 & -0.27 \\
\hline$n$ & 4.07 & $2.94^{*}$ & $3.57^{*}$ & $2.58^{*}$ & 1.82 \\
\hline$J_{k}\left(\mathrm{~mA} \cdot \mathrm{cm}^{-2}\right)$ & -115.2 & -29.2 & -8.7 & -13.6 & -2.2 \\
\hline$k\left(\mathrm{~cm} \cdot \mathrm{s}^{-1}\right)$ & 0.285 & 0.100 & 0.024 & 0.053 & 0.012 \\
\hline
\end{tabular}
different ligand-stabilized AuNPs at $-1.00 \mathrm{~V}$, obtained from Plots in Fig. S5.

The K-L equations model considers that, at high overpotential, the electrolytic process is controlled by mass transport (i.e. $J_{k}$ tends to infinity), and therefore, $J \sim J_{L}$. Thus, the current density must show a plateau (i. e., a constant value and equal to $J_{L}$ ) at high overpotential. The expected behaviour was clearly observed for Bare-GC and AuNPs@Citrate at E <-0.90 V. However, AuNPs@CTAB, AuNPs@PSs and AuNPs@MUA displayed a different behavior, where the resulting current density monotonically increased for AuNPs@PSS and AuNPs@MUA, whilst it decreased for AuNPs@CTAB (Fig. 6A). As a consequence of this, the $\mathrm{K}-\mathrm{L}$ equations model is not applicable to those systems probably due to the electrochemical processes are more complex than in the mentioned model. The calculation of the overall number of electrons transferred in oxygen reduction for these ligand-coated AuNPs cannot be made with the mentioned equations, and the $n$ values might be considered as apparent, Table 1 . This finding is supported by the crossing of the K-L plots for the CTAB, PSS and MUA ligand-stabilized AuNPs that can be considered as an inconsistency of the K-L model, 
given that no crossing is expected when the process is purely controlled by mass transport (i.e. at rotating conditions), see Fig. 6B.

Additionally, Pb-UPD analysis after ORR measurements were performed on the different ligand-stabilized AuNPs (Figure 4B). Surprisingly, significant faceting transformation can be observed for AuNPs@Citrate with a clear tendency to acquire (111) symmetry, which is the one of the lowest surface energies. $^{48}$

\section{HER analysis}

The influence of the capping ligands on the electrocatalytic properties of AuNPs for HER in $\mathrm{N}_{2}$-saturated acidic solution was also assessed. Figure 7A shows the resulting linear sweep voltammograms (LSVs) for HER. Table 2 summaries the values of onset overpotential $(\eta)$, the $\eta$ at 10 and $50 \mathrm{mV} \cdot \mathrm{cm}^{-2}$ obtained from LSVs in Fig. 7A. An evident effect of the capping ligand can be observed for HER similarly to ORR, obtaining the best performance for the citrate-stabilized AuNPs. The Tafel slopes were calculated from Fig. 7B. The resulting overpotential $(\eta)$ at -10 $\mathrm{mV} \cdot \mathrm{cm}^{-2}$ and Tafel slope values for AuNPs@Citrate were quite similar to those reported recently by Tran et al. for $350 \mu \mathrm{g}-$ Au-NPs-13nm/FTO electrode. ${ }^{43}$ In addition, the obtained catalytic activity is only 2-fold lower than the best reported for a Pt-based electrocatalyst. ${ }^{50}$ The mechanistic pathway in the HER process for the different ligand-stabilized AuNPs was examined by RDVs measurements (Fig. S6). The hydrogen evolution rate increases at the rotation rate of $1600 \mathrm{rpm}$, further confirming that for AuNPs-based catalysts the mass diffusion of proton is limiting stage, and thus, the Heyrovsky step may be the rate-determining step (RDS): ${ }^{51}$

$$
\mathrm{Au}-\mathrm{H}+\mathrm{H}_{3} \mathrm{O}^{+}+\mathrm{e}^{-} \leftrightarrow \mathrm{H}_{2}+\mathrm{H}_{2} \mathrm{O}+\mathrm{Au}
$$

Next, durability test of the different ligand-stabilized AuNPs was performed by chronopotenciometry at a current density of $-10 \mathrm{~mA} \cdot \mathrm{cm}^{-2}$ for $12 \mathrm{~h} .{ }^{52}$ As can been observed in Fig. S7A, in all cases the overpotential remained almost constant for $12 \mathrm{~h}$, demonstrating the good electrochemical stability of AuNPs. In order to further confirm the good long-term stability of the different AuNPs toward HER, linear sweep voltammograms were performed after durability test. Fig. S7B shows that AuNPs not only maintained the electrocatalytic capabilities for AuNPs@CTAB and AuNPs@MUA, but also enhanced for AuNPs@Citrate and AuNPs@PSS.The latter improvement could be associated with the ligand loss during the long-term measurements because it was only observed for AuNPs with the weaker Au-ligand bonding interactions.

Finally, Turn Over Frequency (TOF) and Faradaic Efficiency (FE) values were calculated for AuNPs@Citrate. The resulting TOF value at - $200 \mathrm{mV}$ was $0.1 \mathrm{~s}^{-1}$, which was obtained from Fig. $7 \mathrm{~A}$ and using the equation (5) that considers two electrons to produce one $\mathrm{H}_{2}$ molecule: 52

$$
\text { TOF }=J \cdot A / 2 \cdot F \cdot m \quad(5)
$$

Where $J$ is the current density at a given overpotential $(\eta=-200$ $\mathrm{mV}), \mathrm{A}$ is the surface area of the electrode $\left(0.2 \mathrm{~cm}^{-2}\right), F$ is the Faraday constant (a value of $96485 \mathrm{C} / \mathrm{mol}$ ), and $\mathrm{m}$ is the number of moles of gold on the electrode $\left(1.75 \cdot 10^{-8}\right.$ moles de $\left.\mathrm{Au}\right)$. Whilst to calculate the FE value for AuNPs@Citrate, chronoamperometry measurements at a constant potential ( $E$ $=-270 \mathrm{mV}$ ) for two specific times were carried out, and the volumes of the generated hydrogen were measured by water displacement method. ${ }^{53}$ Then, FE values were estimated by comparing the experimental volume of produced hydrogen with the theoretically calculated for the charge passed (e.g. considering a conversion of $100 \%, 1 \mathrm{C}$ should produce $0.116 \mathrm{~mL}$ of $\mathrm{H}_{2}$ ), using the following equation:

$$
\mathrm{FE}=2 \cdot F \cdot n_{\mathrm{H} 2} / Q \quad(6)
$$

Where $\mathrm{n}_{\mathrm{H} 2}(\mathrm{~mol})$ is the total amount of hydrogen produced and $Q(C)$ is the total amount of charge passed through the cell. As it can be observed in Fig. SX, the resulting faradaic yield of AuNPs@Citrate for HER was nearly 100\%.

\section{Post ORR and HER analysis}

XPS analysis of the different ligand-stabilized AuNPs were performed after ORR and HER measurements (Fig. SX-SZ). Overall, the oxidation state of gold cores does not vary after both cathodic processes, whilst the ligands display two different behaviours: On the one hand, AuNPs@Citrate and AuNPs@PSS (i.e. Au-O bonds) show significant loss of their respective surface coverage of ligands. And on the other hand, AuNPs@CTAB (i.e.Au-N bond) y AuNPs@MUA (i.e. Au-S bond) almost do not change. This difference can be attributed to the distinct bonding interaction forces between Au-core and each
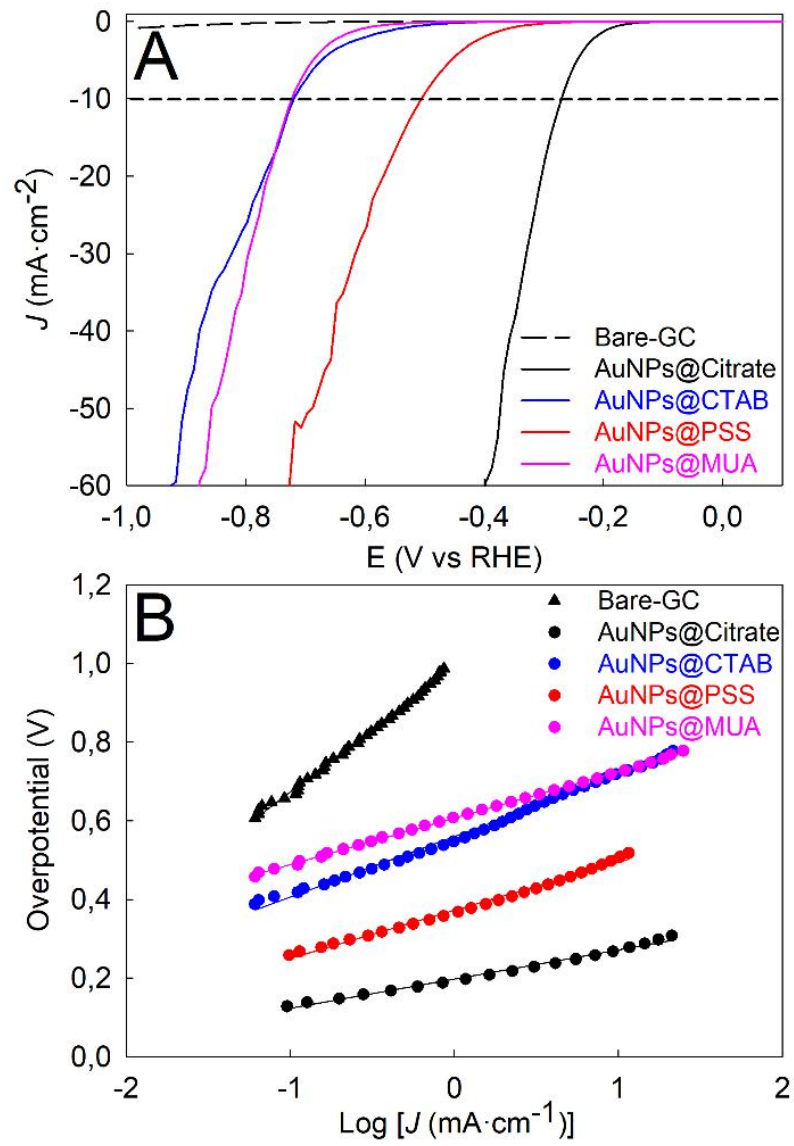

Fig. 7 (A) Linear sweep voltammograms (LSVs), without iR-correction, of GC electrodes modified with different ligand-stabilized AuNPs in $\mathrm{N}_{2}$ saturated $0.5 \mathrm{M} \mathrm{H}_{2} \mathrm{SO}_{4}$. Scan rate: $2 \mathrm{mV} \cdot \mathrm{s}^{-1}$, and $(\mathrm{B})$ the resulting Tafel polarization plots 
stabilizing ligand (i.e. Au-S and Au-N bonds are stronger than Au-O interactions).

Table 2. HER electrochemical parameters for HER of the GC electrodes modified with different ligand-stabilized AuNPs, obtained from Plots in Fig. 7.

\begin{tabular}{|c|c|c|c|c|}
\hline $\begin{array}{c}\text { HER } \\
\text { activity }\end{array}$ & AuNPs@Citrate & AuNPs@CTAB & AuNPs@PSS & AuNPs@MUA \\
\hline $\begin{array}{c}\text { Onset } \\
\text { Overpotential } \\
\eta(\mathrm{V})\end{array}$ & -0.12 & -0.40 & -0.25 & -0.47 \\
\hline $\begin{array}{c}\eta(\mathrm{V}) \text { at } 10 \\
\mathrm{mV} \cdot \mathrm{cm}^{-2}\end{array}$ & -0.27 & -0.72 & -0.50 & -0.72 \\
\hline $\begin{array}{c}\eta(\mathrm{V}) \text { at } 50 \\
\mathrm{mV} \cdot \mathrm{cm}^{-2}\end{array}$ & -0.37 & -0.90 & -0.69 & -0.86 \\
\hline $\begin{array}{c}\text { Tafel slope } \\
\left(\mathrm{mV} \cdot \mathrm{dec}^{-1}\right)\end{array}$ & 74 & 154 & 122 & 118 \\
\hline
\end{tabular}

\section{Conclusions}

For the first time ever, non-doped chemically synthesized AuNPs with suitable bi-functional electrocatalytic performance for ORR and HER was reported. The resulting catalytic parameters of AuNPs@Citrate were quite similar to the one obtained for another recently reported bifunctional electrocatalysts (see Table S1).

In addition, the drastic influence of the Au-ligand interface on the electrocatalytic performance for both cathodic reactions was demonstrated. Both the metal-ligand bonding interaction force and the degree of surface coverage are dependent parameters of the organic ligand type that affect not only to the available surface of metal for the reactant adsorption (e.g. $\mathrm{O}_{2}$ and $\mathrm{H}^{+}$for ORR and $\mathrm{HER}$, respectively) but also to facilitate the ligand displacement. Therefore, the capping ligand plays an important role on the resulting electrocatalytic performances of nanoparticles-based electrocatalysts. In this direction, the outstanding electrocatalytic properties of the AuNPs@Citrate towards ORR and HER reactions were attributed to the ability of this molecule to allow the efficient adsorption of $\mathrm{O}_{2}$ and hydronium ions onto the nanoparticle's surfaces. The small dimensions of citrate molecules combined with both their weak attachment to the Au-cores and the high overall negative surface charge density may strongly increase the number of $\mathrm{O}_{2}$ molecules and hydronium species at the electrochemical interfaces and in turn decrease the activation energy of the electrocatalytic processes.

The chemical versatility of citrate for allowing successful ligand exchange processes, based on simple concentration gradients, has been proven. The proposed procedure paves the way for exploring other organic and organic/inorganic ligand derivatives for tuning the electrocatalytic performance of bifunctional Au-cores. Our approach can be readily extrapolated to other metal-based nanoelectrocatalysts. This work suggests that easy, on-demand chemical functionalization of the metallic surface of nanostructures can finely adjust the electrocatalytic activity in renewable energy applications.

\section{Conflicts of interest}

There are no conflicts to declare.

\section{Acknowledgements}

Support from the Ministry of Economy and Competitiveness of Spain is acknowledged through the MANA project CTQ201783961-R. J.J.G.-C. acknowledges the Ministry of Economy and Competitiveness for a "Ramon y Cajal" contract (\#RyC-201414956). M. C. thanks the "Plan Propio de Investigación" from the Universidad de Córdoba (UCO) and the "Programa Operativo de fondos FEDER Andalucía" for its financial support through a postdoctoral contract (Modality 5.2.A). The publication has been prepared with support from RUDN University Program 5-100. Rafael Luque gratefully acknowledges MINECO for funding project CTQ2016-78289-P, co-financed with FEDER funds. Enrique Rodríguez-Castellón acknowledges Ministerio de Ciencia, Innovación y Universidades of Spain for funding project RTI2018-099668-BC22 and FEDER funds. Alain R. Puente-Santiago gratefully acknowledge MINECO for their research contracts associated to the aforementioned project, and to the Research Program of the UCO for its financial support through a postdoctoral contract (Modality 5.1). They also thank Prof. Dr. J.M. Rodríguez-Mellado of the Department of Physical Chemistry and Applied Thermodynamics at the UCO for kind access to the rotating disk equipment.

\section{Notes and references}

1 X. Yuan, L. Zhang, L. Li, H. Dong, S. Chen, W. Zhu, C. Hu, W. Deng, Z.-J. Zhao, J. Gong, J. Am. Chem. Soc., 2019, 141, 47914794.

2 J. H. Montoya, L. C. Seitz, P. Chakthranont, A. Vojvodic, T.F. Jaramillo, J. K. Nørskov, Nat. Mat., 2017, 16, 70-81.

3 Y. P. Zhu, C. Guo, Y. Zheng, S.-Z. Qiao, Acc. Chem. Res., 2017, 50, 915-923.

4 M. Shao, Q. Chang, J.-P. Dodelet, R. Chenitz, Chem. Rev., 2016 116, 3594-3657.

5 X.-B. Han, X.-Y. Tang, Y. Lin, E. Gracia-Espino, S.-G. Liu, H.-W. Liang, G.-Z. Hu, X.-J. Zhao, H.-G. Liao, Y.-Z. Tan, T. Wagberg, S.Y. Xie, L.-S. Zheng, J. Am. Chem. Soc., 2019, 141, 232-239.

6 C. L. Bentley, M. Kang, P. R. Unwin, J. Am. Chem. Soc., 2019, 141, 2179-2193.

7 J.-C. Dong, X.-G. Zhang, V. Briega-Martos, X. Jin, J. Yang, S. Chen, Z.-L. Yang, D.-Y. Wu, J. M. Feliu, C. T. Williams, Z.-Q. Tian, J.-F. Li, Nat. Energy, 2019, 4, 60-67.

8 A. M. Gómez-Marín, J. M. Feliu, E. Ticianelli, ACS Catal., 2019, 9, 2238-2251.

9 K. Kusada, D. Wu, T. Yamamoto, T. Toriyama, S. Matsumura, W. Xie, M. Koyama, S. Kawaguchi, Y. Kubotag, H. Kitagawa, Chem. Sci., 2019, 10, 652-656.

10 L. Bu, Q. Shao, B. E, J. Guo, J. Yao, X. Huang, J. Am. Chem. Soc., 2017, 139, 9576-9582.

11 B. Wu, N. Zheng, Nano Today, 2013, 8, 168-197.

12 R.-Y. Zhong, K.-Q. Sun, Y.-C. Hong, B.-Q. Xu, ACS Catal., 2014, 4, 3982-3993.

13 L. Lu, S. Zou, Y. Zhou, J. Liu, R. Li, Z. Xu, L. Xiao, J. Fan, Catal. Sci. Technol., 2018, 8, 746-754.

14 E. L. Clark, S. Ringe, M. Tang, A. Walton, C. Hahn, T. F. Jaramillo, K. Chan, A. T. Bell, ACS Catal., 2019, 9, 4006-4014. 
15 T. Su, Q. Shao, Z. Qin, Z. Guo, Z. Wu, ACS Catal., 2018, 8, 2253 2276.

16 K. O. Aruda, M. Tagliazucchi, C. M. Sweeney, D. C. Hannah, E. A. Weiss, Phys. Chem. Chem. Phys., 2013, 15, 7441-7449.

17 W. Lewandowski, M. Fruhnert, J. Mieczkowski, C. Rockstuhl, E. Górecka, Nat. Commun., 2015, 6, 6590.

18 P. Sonström, D. Arndt, X. Wang, V. Zielasek, M. Bäumer, Angew. Chem. Int. Ed., 2011, 50, 3888-3891.

19 S. Elbasuney, Appl. Surf. Sci., 2017, 409, 438-447.

20 S. Zhou, D. Huo, S. Goines, T.-H. Yang, Z. Lyu, M. Zhao, K. D. Gilroy, Y. Wu, Z. D. Hood, M. Xie, Y. Xia, J. Am. Chem. Soc., 2018, 140, 11898-11901.

21 Z.-Y. Zhou, X. Kang, Y. Song, S. Chen, J. Phys. Chem. C, 2012, 116, 10592-10598.

22 B. Genorio, R. Subbaraman, D. Strmenik, D. Tripkovic, V. R. Stamenkovic, N. M. Markovic, Angew. Chem. Int. Ed., 2011, 50, 5468-5472.

23 P. N. Duchesne, Z. Y. Li, C. P. Deming, V. Fung, X. Zhao, J. Yuan, T. Regier, A. Aldalbahi, Z. Almarhoon, S. Chen, D.-e. Jiang, N. Zheng, P. Zhang, Nat. Mat., 2018, 17, 1033-1039.

24 M. Babucci, C.-Y. Fang, J. E. Perez-Aguilar, A. S. Hoffman, A. Boubnov, E. Guan, S. R. Bare, B. C. Gates, A. Uzun, Chem. Sci., 2019, 10, 2623-2632.

25 F. P. Da Silva, J. L. Fiorio, L. M. Rossi, ACS Omega, 2017, 2, 6014-6022.

26 L. Jin, B. Liu, S. S. Duay, J. He, Catalysts, 2017, 7, 44.

27 Y. Zhao, L. Tao, W. Dang, L. Wang, M. Xia, B. Wang, M. Liu, F. Gao, J. Zhang, Y. Zhao, Small, 2019, 15, 1900288.

28 M. Guo, H. Li, Y. Ren, X. Ren, Q. Yang, C. Li, ACS Catal., 2018, 8, 6476-6485.

29 G. Chen, C. Xu, X. Huang, J. Ye, L. Gu, G. Li, Z. Tang, B. Wu, H. Yang, Z. Zhao, Z. Zhou, G. Fu, N. Zheng, Nat. Mat., 2016, 15 564-569.

30 J. Zhang, L. D. Ellis, B. Wang, M. J. Dzara, C. Sievers, S Pylypenko, E. Nikolla, J. W. Medlin, Nat. Catal., 2018, 1, 148155.

31 Z. W. Seh, J. Kibsgaard, C. F. Dickens, I. Chorkendorff, J. K. Nørskov, T. F. Jaramillo, Science, 2017, 355, eaad4998.

32 D. Mukherjee, P. M. Austeria, S. Sampath, ACS Appl. Energy Mater., 2018, 1, 220-231.

33 S. Ghosh, R. N. Basu, Nanoscale, 2018, 10, 11241-11280.

34 J. Turkevich, P. C. Stevenson, J. Hillier, Discuss. Faraday Soc., 1951, 55-75.

35 D. Alba-Molina, D. Rodríguez-Padrón, A. R. Puente-Santiago, J. J. Giner-Casares, M. T. Martín-Romero, L. Camacho, L. O. Martins, M. J. Muñoz-Batista, M. Cano, R. Luque, Nanoscale, 2019, 11, 1549-1554.

36 N. G. Bastús, J. Comenge, V. Puntes, Langmuir, 2011, 27, 11098-11105.

37 C. M. Cova, A. Zuliani, A. R. Puente Santiago, A. Caballero, M. J. Muñoz-Batista, R. Luque, J. Mater. Chem. A, 2018, 6, 2151621523.

38 J.-W. Park, J. S. Shumaker-Parry, J. Am. Chem. Soc., 2014, 136, 1907-1921.

39 J. G. Mehtala, D. Y. Zemlyanov, J. P. Max, N. Kadasala, S. Zhao, A. Wei, Langmuir, 2014, 30, 13727-13730.

40 B. M. Amoli, S. Gumfekar, A. Hu, Y. N. Zhou, B. Zhao, J. Mater. Chem., 2012, 22, 20048-20056.

41 L. Su, S. Hu, L. Zhang, Z. Wang, W. Gao, J. Yuan, M. Liu, Small, 2017, 13, 1602809.

42 M. Murdoch, G. Waterhouse, M. A. Nadeem, J. B. Metson, M. A. Keane, R. F. Howe, J. Llorca, H. Idriss, Nat. Chem., 2011, 3 489-492.

43 T. D. Tran, M. T. T. Nguyen, H. V. Le, D. N. Nguyen, Q. D. Truong, P. D. Tran, Chem. Commun., 2018, 54, 3363-3366.

44 F. Lu, Y. Zhang, S. Liu, D. Lu, D. Su, M. Liu, Y. Zhang, P. Liu, J. X Wang, R. R. Adzic, O. Gang, J. Am. Chem. Soc., 2017, 139 7310-7317.
45 D. Alba-Molina, A. R. Puente Santiago, J. J. Giner-Casares, M. T. Martín-Romero, L. Camacho, R. Luque, M. Cano, J. Phys. Chem. C, 2019, 123, 9807-9812.

46 H. Al-Johani, et al. Nat. Chem., 2017, 9, 890-895.

47 J. Pérez-Juste, I. Pastoriza-Santos, L. M. Liz-Marzán, P. Mulvaney, Coord. Chem. Rev., 2005, 249, 1870-1901.

48 J. Kumar, K. G. Thomas, J. Phys. Chem. Lett., 2011, 2, 610-615.

49 H. Cheng, L. Yang, Y. Jiang, Y. Huang, Z. Sun, J. Zhang, T. Hu, Z. Pan, G. Pan, T. Yao, Q. Bian, S. Wei, Nanoscale, 2013, 5, 1179511800.

50 Z. Chen, D. Cummins, B. N. Reinecke, E. Clark, M. K. Sunkara, T. F. Jaramillo, Nano Lett., 2011, 11, 4168-4175.

51 C. Costentin, M. Robert, J.-M. Savéant, Chem. Rev., 2010, 110 PR1-PR40.

52 F. Song, X. Hu, Nat. Commun., 2014, 5, 4477-4486.

53 Z. Huang, Z. Chen, Z. Chen, C. Lv, M. G. Humphrey, C. Zhang, Nano Energy, 2014, 9, 373-382. 